die der Betreffende vielleicht überhaupt erwerbsunfähig würde. Er muß also zum dauernden, wenn auch nicht täglichen $\mathrm{Ge}$. brauch von Schlafmitteln seine Zuflucht nehmen. Die in solchen Fällen z. B. oft gegebenen Veronaltabletten sowie die in neuerer Zeit von verschiedenen Seiten empfohlenen Luminaltabletten sind leider sehr teuer. Nimmt man als Normaldosis für Veronal $0,5 \mathrm{~g}$, für Luminal $0,3 \mathrm{~g}$ an, so kostet die Einzelgabe nach

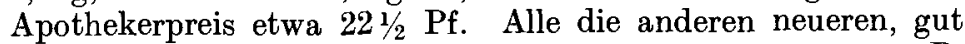
wirkenden Schlafmittel sind wohl sämtlich noch teurer, z. B. das Adalin, dessen Einzeldosis sich auf 35-50 Pf. stellt.

Infolge dieses hohen Preises der Originalpräparate sind wir leider nicht selten gezwungen, zu billigeren Ersatzpräparaten, beispielsweise des Veronals oder des Medinals, d.h. zur Diäthylbarbitursäure bzw. zu dessen Natriumsalz greifen zu müssen. Doch ist man bei derartigen Ersatzmitteln nicht immer sicher, daß das betreffende Produkt von einer vollkommen einwandfreien Provenienz ist. Es ist uns daher mit billigeren Originalpräparaten mehr gedient.

Nun sind zwar Sulfonal und Trional wohlfeiler, indes darf man diese Präparate auf die Dauer nicht anwenden wegen der Gefahr der Schädigung der Nieren. Es blieben also nur "das Chloralhydrat und das Paraldehyd oder ähnliches, die aber wegen ihres widerlichen Geruches und schlechten Geschmackes von etwas empfindlicheren Kranken - und um solche handelt es sich hier doch meistenteils - von vornherein perhorresziert werden.

Es wurde uns nun von der Gesellschaft für Chemische Industrie in Basel vor einiger Zeit ein neues Schlafmittel, DialCiba genannt, zu Versuchszwecken zur Verfügung gestellt. Dieses Mittel hat sich bei der von uns vorgenommenen eingehenden Prüfung als ein durchaus brauchbares, in gewissen Fällen sogar den bekannten Schlafmitteln überlegenes Präparat erwiesen und hat überdies noch den uns besonders willkommenen Vorzug eines billigen Originalpräparates. Das Dial-Ciba ist bereits in wesentlich geringeren Dosen wirksam als Veronal und Medinal. Als hypnogene Dosis bei nicht komplizierten Fällen von Schlaflosigkeit hat sich uns $0,1-0,15 \mathrm{~g}$ (d. h. 1 bis $11 / 2$ Originaltabletten à $0,1 \mathrm{~g}^{1}$ ) Dial ergeben. Der Preis dieser Dialdosis stellt sich auf 10-15 Pf. Auf Anfrage bei der Fabrik erfahren wir übrigens, daß auch Packungen mit 250 bzw. 1000 Tabletten speziell für Krankenhäuser und Heilanstalten geliefert werden und sich der Preis einer Tablette bei Bezug dieser großen Packungen noch erheblich billiger stellt.

Chemisches: Nach Angabe der Fabrik ist Dial-Ciba chemisch Diallylbarbitursäure (Diallylmalonylharnstoff) von der Formel

$$
\mathrm{CH}_{2}=\mathrm{CH}-\mathrm{CH}_{2}>\mathrm{CH}<\mathrm{CO}-\mathrm{NH}>\mathrm{NH}
$$

Dial-Ciba steht also in bezug auf seine Konstitution mit Ausnahme seiner ungesättigten Natur (zwei Doppelbindungen) den bekannten, als Schlafmittel benutzten Derivaten der Barbitursäure, wie Veronal (Diäthylbarbitursäure), Luminal (Phenyläthylbarbitursäure) etc. nahe.

Dial-Ciba kristallisiert in Form weißer, glänzender Blättchen vom Nervenkranke in Berlin-Buchholz.

\title{
Ueber Dial-Ciba (Diallylbarbitursäure), ein neues Hypnotikum.
}

\section{Von Dr. W. Zuelchanr, II. Arzt.}

Es mag den Anschein haben, als ob die jüngste Zeit uns gerade an Schlafmitteln soviel Neues gebracht hat, da $B$ irgendein fühlbarer Mangel nicht bestände, und daß also ein Bedürfnis nach einem weiteren nicht im geringsten mehr vorläge, und dieses besonders auch deswegen, weil es ja hauptsächlich in den allerletzten Jahren gelungen ist, so verschiedenartige Präparate dieser Art herzustellen, daß man mit ihnen eigentlich jede gewünschte Wirkung von der leichtesten sedativen bis hinauf zur schwersten hypnotischen leicht zu erzielen imstande ist. Betrachtet man es indessen von einer anderen Seite, die zwar gerade bei einem Medikament niemals die allein ausschlaggebende sein dürfte, indessen trotzdem recht häufig schwer ins Gewicht fällt, so wird man bald darüber anderer Ansicht werden. Nehmen wir beispielsweise nur folgenden Fall: Ein Neurastheniker der minderbemittelten Stände leidet an der mit dieser Neurose ja meist verbundenen hartnäckigen Agrypnie, soda $B$ der Arzt gezwungen ist, ihm seine an sich schon geschwächte Arbeitskraft wenigstens dadurch noch leidlich zu erhalten, daß er für eine genügende Nachtruhe sorgt, ohne Schmelzpunkt 170-171. Die Löslichkeit in kaltem Wasser ist gering, größer in heißem Wasser. In Azeton und Essigäther ist Dial-Ciba leicht löslich, etwas schwerer in Alkohol und Aether.

Dial-Ciba ist eine sehr schwache Säure. Seine konzentrierte wäßrige Lösung reagiert auf Lackmus schwach sauer. Aetznatron oder Sodalösung (nicht Bikarbonat) lösen Dial unter Bildung eines leicht wasserlöslichen, stark alkalisch reagierenden Natriumsalzes von beschränkter Beständigkeit. Gegenüber Wasser und verdünnten Säuren ist DialCiba vollkommen resistent. Dagegen wird Dial infolge seines ungesättigten Charakters von Oxydationsmitteln (z. B. von Permanganat) angegriffen.

Pharmakologisches: Mit Dial-Ciba wurden im Pharmakologischen Laboratorium der Gesellschaft für Chemische Industrie in Basel eingehende Untersuchungen angestellt. Als Versuchstiere dienten Hunde, Kaninchen und Katzen. Das Dial-Ciba erwies sich dabei als erheblich wirksamer als die übrigen Derivate der Barbitursäure, indem es in erheblich kleineren Dosen dieselbe Tiefe der Narkose bewirkte wie die genannten Schlafmittel. Die Dauer der Narkose war aber bei sonst analog wirkenden Gaben von Dial kürzer als nach den erwähnten anderen Präparaten, und das Erwachen aus der Narkose war insbesondere bei Hunden nicht mit so lang dauernden posthypnotischen Erscheinungen verbunden wie bei der Diäthylbarbitursäure. Ferner wurden bei lang dauernder Verabreichung von Dial nie Kumulationserscheinungen

1) $\mathrm{Zu}$ bemerken ist, daß die Dialtablette à $0,1 \mathrm{~g}$ Dial in der Mitte gut gekerbt ist, sodaß sich die Dosis von $1 \frac{1}{2}$ Tablette $=0,15 \mathrm{~g}$ wirk samer Substanz leicht herstellen läßt. 
beobachtet. Reizwirkungen auf den Verdauungskanal oder Wirkungen auf das Blut, die auf eine Abspaltung der Allylgruppe hätten schließen lassen, wurden nie beobachtet.

Die verhältnismäßig kurze Dauer der Dialnarkose, das Wegbleiben von stärkeren posthypnotischen Symptomen und das Fehlen von Kumulationserscheinungen ist offenbar darauf zurückzuführen, daß Dial (im Gegensatz zu anderen synthetischen Schlafmitteln) im Organismus vollkommen verbrannt wird. Es wurde nämlich bei Versuchen an Hunden und Kaninchen festgestellt, daß weder bei chronischer Verabreichung großer Dialdosen noch bei einmaliger Darreichung der tödlichen Dosis unverändertes Dial im Urin ausgeschieden wird, während von verfütterter Diäthylbarbitursäure bis zu $80 \%$ im Urin nachweisbar sind.

Dial wird also nicht als solches im Harn ausgeschieden. Es wird auch nicht als Abbauprodukt eine andere Säure ausgeschieden, die noch Biuretreaktion liefert (etwa Dimethylbarbitursäure). Offenbar bietet hier die Doppelbindung einen Angriffspunkt für den Abbau.

Wir haben nun die uns von der Fabrik freundlichst zur Verfügung gestellten Quanten des Präparates an unserer Anstalt zu Versuchen bei den verschiedensten Krankheitsformen verwandt, und ich möchte unsere Erfahrungen über seine Wirkungsweise im Folgenden des näheren darlegen. Unsere Versuche erstrecken sich auf etwa 800 Einzelgaben, die sich auf etwa 25 Fälle bei dauerndem Gebrauch und etwa ebensoviele bei gelegentlicher Medikation verteilen. Man muß bezüglich der Anwendungsweise des Dials notwendig unterscheiden zwischen den Neurosen, Neurasthenie, Hysterie und ähnlichen mit Schlaflosigkeit verbundenen leichteren nervösen Zuständen und ausgesprochenen Psychosen. Ich wende mich zunächst der Besprechung der ersteren zu.

Um lediglich sedative Wirkungen zu erreichen, wird man in diesen Fällen in der Regel wohl von der Darreichung von ausgesprochenen Hypnotica absehen, weil sich derartige Kranke in den Sanatorien ja meist tagsüber außer Bett befinden und dann diese stärker wirkenden Mittel zweifellos Störungen des allgemeinen Wohlbefindens bewirken würden, da sie schließlich, wenn auch in noch so kleinen Dosen verabreicht, doch ein gewisses Schlafbedürfnis auslösen dürften; man wird vielmehr mit den leichteren Sedativmitteln wie etwa Brom- oder Valerianapräparaten auszukommen suchen. Es kann sich hier also nur darum handeln, für die Nacht Schlaf $\mathrm{zu}$ erzeugen. Um diesen Zweck zu erreichen, haben wir bei folgenden Krankheitsformen Dial verabreicht:

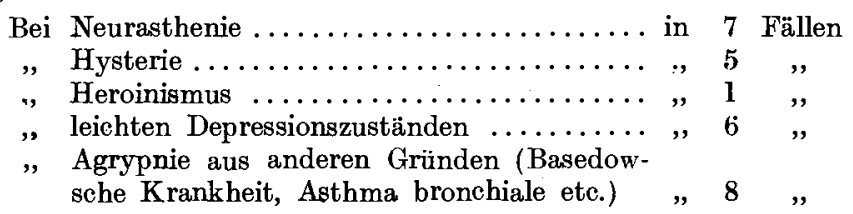

Wir haben bei diesen Erkrankungen meist zunächst $0,1 \mathrm{~g}$ Dial, d. i. eine Tablette, versucht, und in leichteren Fällen häufig schon mit dieser kleinen Dosis einen befriedigenden Schlaf erzielt. Eine Anzahl unserer Patientinnen allerdings reagierte hierauf noch nicht genügend. Diesen wurden dann am nächsten Abend 2 Tabletten $(0,2 \mathrm{~g})$ gegeben, und es wurde mit dieser Dosis nahezu ausnahmslos ein fester, meist die ganze Nacht hindurch andauernder Schlaf hervorgerufen. Ja, einige Kranke klagten sogar bisweilen schon nach dieser Gabe am nächsten Tage noch über eine leichte Schläfrigkeit, sodaß wir als die Normaldosis in ähnlichen Fällen $0,1-0,15$ bis höchstens 0,2 Dial, also $1-1 \frac{1}{2}-2$ Tabletten, annehmen würden. Nur ganz ausnahmsweise kamen wir auch mit 2 Tabletten noch nicht aus, beispielsweise bei dem oben erwähnten Fall von Heroinismus.

Die Patientin litt nach der Entziehung unter einer so hartnäckigen Schlaflosigkeit (übrigens bei ihr wahrscheinlich eine hereditäre Veranlagung, denn auch die Mutter soll Schlafmittel in unglaublicher Menge nehmen), daß sie hierdurch fast zur Verzweiflung gebracht wurde. Alle Schlafmittel waren schon vergeblich durchprobiert worden I,0 Medinal beispielsweise hatte bei ihr nicht die geringste Wirkung. Da verabreichten wir ihr eines Abends 0,3 Dial. Am nächsten Morgen war sie ganz gegen ihre Gewohnheit in der besten Stimmung und erklärte, eine so gute Nacht habe sie seit Monaten nicht gehabt.

Ueber unangenehme Nebenwirkungen oder Nachwirkungen, wie Benommenheit $u$. a. hatte sie nicht zu klagen. Ueberhaupt haben wir solche nach der Darreichung von Dial selbst in relativ hohen Dosen oder bei lange fortgesetztem Gebrauche so gut wie nie gesehen. Allerdings wurde hin und wieder von sehr empfindlichen Frauen über ganz leichtes Schwindelgefühl am Morgen kurz nach dem Verlassen des Bettes geklagt, dieses verlor sich aber stets nach Verlauf von ganz kurzer Zeit. Auch schädliche Wirkung auf die Nieren konnten wir nie beobachten.

Wir haben einer ganzen Reihe von Patientinnen monatelang, höchstens mit ganz kurzen Unterbrechungen, täglich Dial verabreicht unter dauernder Kontrolle des Urins, ohne jemals Albumen nachweisen zu können.

Der Schlaf tritt nach fast übereinstimmender Schätzung aller mit Dial behandelter Kranker etwa $1 / 2-3 / 4$ Stunden nach dem Einnehmen des Mittels ein, ist fest, meist traumlos und erquickend, ähnlich dem natürlichen, nicht durch Medikamente erzeugten.

Was nun zweitens die Anwendung des Mittels bei ausgesprochenen Psychosen betrifft, so haben wir auch bei ihnen an einer Reihe verschiedener Krankheitsformen Versuche angestellt, und hier ebenso, wie ich gleich vorwegnehme, mit durchaus befriedigenden Erfolgen.

Das Mittel wurde angewandt in

\begin{tabular}{|c|c|c|c|}
\hline 6 Fällen & von & Dementia praecox & \\
\hline $2 \quad$, &, & Dementia paralytica & \\
\hline ", & , & zirkulärem Irresein in beiden & Phasen \\
\hline ," & $\therefore$ & Angstpsychose & \\
\hline , & " & Dementia senilis & \\
\hline , & ," & $\begin{array}{l}\text { anderen Krankheitsformen } \\
\text { schöpfung etc.) }\end{array}$ & (Korsakow, \\
\hline
\end{tabular}

Zunäclist muß hierbei ein Unterschied gemacht werden, ob man nämlich eine sedative Wirkung erzielen will oder eine ausgesprochen schlafmachende.

Einer Präkoxkranken, die sonst den ganzen Tag höchst unruhig umherläuft, fortwährend schwatzt, häufig aufschreit, dauernd stereotype Bewegungen macht, die anderen Kranken und jeden, der sonst auf die Abteilung kommt, dadurch belästigt, daß sie ihn anfaßt, umarmt oder ähnliches, wurden dreimal täglich 0,1 Dial verabreicht. Die Wirkung war eklatant. Die Kranke saß ruhig, fast ohne sich zu bewegen, auf ihrem Platze und machte sich den ganzen Tag über für niemanden unangenehm bemerkbar. In einem anderen Falle von Dementia paranoides mit sehr stark gefühlsbetonten, abundanten Größenvorstellungen und daraus resultierenden, zienlich heftigen Erregungszuständen wurde wiederholt während der letzteren 0,2 Dial verabreicht. Nach relativ kurzer Zeit, etwa einer halben Stunde, beruhigte sich die Kranke regelmäßig, setzte sich still in eine Sofaecke und kämpfte sichtlich mit dem Schlafe.

Aehnliche, wenn auch vielleicht nicht immer ganz so schöne Wirkungen sahen wir auch noch in einer Reihe von anderen Fällen, sodaß wir also die für eine sedative Wirkung geeignete Dosis bei dauernden leichteren Unruhezuständen Geisteskranker $3 \mathrm{mal}$ täglich 1 Tablette annehmen möchten, bei schwereren Erregungszuständen aber eine einmalige oder auch wiederholte Gabe von $0,2 \mathrm{~g}$. Um bei unruhigeren Geisteskranken eine schlafmachende Wirkung $\mathrm{zu}$ erzielen, ist man allerdings gezwungen, gleich mit etwas größeren Gaben zu beginnen, etwa mit $0,2 \mathrm{~g}$. Wenigstens haben wir mit geringeren Mengen als diese keine rechte Wirkung gesehen. Die Dosis von $0,2 \mathrm{~g}$ genügt indessen wohl in den meisten Fällen, um wenigstens für eine Reihe von Stunden während der Nacht Ruhe und Schlaf zu erzeugen.

Besonders möchte ich hier einen der oben angeführten Fälle von Angstpsychose hervorheben. Die Kranke jammerte vor Angst fast unausgesetzt den ganzen Tag, litt an einer nicht beherrschbaren Unruhe warf sich fortwährend im Bett umher, rang verzweifelt die Hände, zupfte an ihren Fingerspitzen und an dem Bettuch, kurz, zeigte das typische Bild der furchtbarsten Angst. Sie hatte nach Angaben ihrer Angehörigen, bevor sie in die Anstalt kam, zu Hause überhaupt nicht mehr geschlafen, war die ganzen Nächte hindurch ruhelos umhergelaufen, durch ihre Angst von Ort zu Ort getrieben. Wir verabreichten ihr am ersten Abend ihres Aufenthaltes in unserer Anstalt, also bevor irgendeine sonstige Kur mit ihr begonnen war, 0,2 g Dial und hatten den Erfolg, $\mathrm{da} B$ sie eine relativ ruhige Nacht verbrachte. Sie schlief zwar nicht ohne Unterbrechungen die ganze Nacht hindurch und jammerte wohl auch zeitweise etwas, sie blieb jedoch im Bett und schlief nach ziemlich kurzer Zeit immer wieder ein.

Auch in den beiden oben genannten, allerdings nicht gerade sehr schweren Fällen von zirkulärem Irresein genügte sowohl in der manischen als auch in der depressiven Phase die Gabe von $0,2 \mathrm{~g}$ vollständig zur Erzielung eines befriedigenden Schlafes. Nur wenige Male kamen wir mit $0,2 \mathrm{~g}$ Dial nicht aus, und 
zwar in zwei Fällen von Dementia praecox und in einem Falle von Paralyse, abgesehen von der oben erwähnten Heroinkranken. Fs handelte sich allerdings in allen drei Fällen um sehr schwer, zeitweise bis zur Tobsucht erregte Kranke. Hier versagten $0,2 \mathrm{~g}$ Dial völlig, während $0,3 \mathrm{~g}$ die Patientinnen deutlich ruhiger stellte und sogar, wenn auch nur vorübergehend, etwas Schlaf bewirkte. Bei diesen Kranken indessen hatte meist auch nicht einmal Morphium-Skopolamin in relativ hohen Dosen (0,009 Skopolamin + 0,02 Morphium) eine nachhaltigere Wirkung. Bis auf $0,4 \mathrm{~g}$ Dial sind wir nur ganz vereinzelt, beispielsweise bei der schon wiederholt erwähnten Heroinkranken hinaufgegangen. Selbst bei Verabreichung dieser Dosis als einmaliger Gabe haben wir keine schädlichen Folgen oder Nachwirkungen gesehen.

Wenn auch nach den obigen Darlegungen das Dial sich uns als ein sehr brauchbares und in seiner Wirkung zuverlässiges Mittel bewährt hat, so haftet ihm dennoch ein Fehler an, der indessen seine Brauchbarkeit nicht wesentlich herabzusetzen imstande sein dürfte, und das ist die relativ schnell eintretende Gewöhnung daran und die dadurch bedingte Verminderung seiner Wirkung, ein Fehler übrigens, der ja in mehr oder minder starkem Grade bei den meisten der uns bekannten Schlafmittel in die Erscheinung tritt. Schon nach etwa sechs bis acht Tagen regelmäßigen Gebrauches, bei manchen Kranken sogar noch früher, kann man in der Regel eine deutliche Abnahme seiner Wirksamkeit konstatieren. Das hat zwar nicht viel zu sagen, wenn man die Medikation mit 1 oder 1/1/2 Tabletten beginnen konnte und damit eine genügende Wirkung erzielte. Man kann dann getrost die Dosis sukzessive auf $2-21 / 2-3$ Tabletten und womöglich noch mehr steigern und braucht erst dann, wenn auch diese Gaben versagen, mit dem Mittel zu wechseln. Unangenehmer wird der Fehler, wenn man gezwungen war, gleich mit zwei Tabletten oder noch mehr zu beginnen. Dann ist man allerdings ziemlich rasch am Ende der Möglichkeit einer weiteren Darreichung angelangt und somit vor die Notwendigkeit gestellt, zeitweise andere Mittel zu geben. Allerdings kann man dann nach einer Pause von etwa drei bis vier Tagen wieder mit der vollen Wirkung des Dials rechnen.

Entsprechend dem besonders in neuerer Zeit oft betonten Grundsatze, da $\beta$ zwei ähnlich wirkende Mittel zusammen sich in ihrer Wirkung nicht nur addieren, sondern potenzieren, haben wir in einer Reihe von Fällen Dial kombiniert mit Codein verabreicht, und zwar besonders dann, wenn das reine Dial infolge von Gewöhnung zu versagen begann.

Wir haben folgende verschiedene Kombinationen versucht:

$$
\begin{array}{ll}
0,1 & \text { Dial }+0,01 \text { Cod. phosph. } \\
0,15 \text { Dial }+0,015 \text { Cod. phosph. } \\
0,2 \text { Dial + 0,02 Cod. phosph. }
\end{array}
$$

Ich kann jedoch nicht sagen, daß der Zusatz von Codein die Wirkung des Dials in den besagten Fällen irgendwie merklich verstärkt hätte. Nur in einem Falle von Agrynie als Folge von Asthma bronchiale mit sehr heftigem Angstgefühl hat uns die Kombination ganz gute Dienste geleistet, jedoch war hier der Erfolg wohl weniger der Kombination der beiden Mittel als vielmehr der Wirkung des Narcoticums allein zuzuschreiben.

Zusammenfassung. Das Dial ist ein Hypnoticum und Sedativum, das sich bezüglich seiner Wirksamkeit den anderen neueren Präparaten dieser Art als durchaus gleichwertig an die Seite stellen läßt, ja sogar in viel kleineren Dosen die gleiche Wirkung entfaltet wie sie, besitzt aber infolge seines billigeren Preises eine bei weitem größere Anwendungṣmöglichkeit. Die geeigneten Dosen sind bei leichteren Fällen von Schlaflosigkeit 1-1 1/2 Tabletten eine halbe Stunde vor dem Zubettgehen, bei schwereren Fällen oder bei Psychosen als Schlafmittel 2-3-4 Tabletten, bei letzteren als Sedativum gegen leichtere Unruhezustände $3 \mathrm{mal}$ täglich eine Tablette, gegen schwerere Erregungszustände 2-3 Tabletten als einzelne Gabe eventuell wiederholt. Sollte sich bei länger dauerndem Gebrauch des Präparates die Wirksamkeit des Dials vermindern, so empfiehlt es sich, das Mittel für einige Tage auszusetzen, da nach einer Pause von wenigen Tagen wieder die volle Wirkung des Dial-Ciba hervortritt.
Schädliche Nebenwirkungen sind bei der angegebenen Dosierung des Präparates nicht zu fürchten. 